PROCEEDINGS OF THE

AMERICAN MATHEMATICAL SOCIETY

Volume 126, Number 10, October 1998, Pages 2895-2902

S $0002-9939(98) 04516-\mathrm{X}$

\title{
SOME THETA FUNCTION IDENTITIES RELATED TO THE ROGERS-RAMANUJAN CONTINUED FRACTION
}

\author{
SEUNG HWAN SON
}

(Communicated by Dennis A. Hejhal)

\begin{abstract}
In his first and second letters to Hardy, Ramanujan made several assertions about the Rogers-Ramanujan continued fraction $F(q)$. In order to prove some of these claims, G. N. Watson established two important theorems about $F(q)$ that he found in Ramanujan's notebooks. In his lost notebook, after stating a version of the quintuple product identity, Ramanujan offers three theta function identities, two of which contain as special cases the celebrated two theorems of Ramanujan proved by Watson. Using addition formulas, the quintuple product identity, and a new general product formula for theta functions, we prove these three identities of Ramanujan from his lost notebooks.
\end{abstract}

\section{INTRODUCTION}

In his notebooks [7], [1, p. 34], Ramanujan defined his general theta function $f(a, b)$ by

$$
f(a, b):=\sum_{k=-\infty}^{\infty} a^{k(k+1) / 2} b^{k(k-1) / 2}, \quad|a b|<1 .
$$

This function has the same generality as the classical theta function $\vartheta(z, q)[11$, pp. 463, 464] After Ramanujan, define $f(-q):=f\left(-q,-q^{2}\right)$.

In his famous letters to Hardy [6, pp. 29, 57], Ramanujan defined the RogersRamanujan continued fraction

$$
F(q):=\frac{1}{1}+\frac{q}{1}+\frac{q^{2}}{1}+\frac{q^{3}}{1}+\ldots, \quad|q|<1,
$$

and evaluated it for certain values of $q$. The following two identities are two of the most important theorems about $F(q)$.

Theorem 1.1. For $|q|<1$,

$$
\frac{1}{F(q)}-q^{1 / 5}-q^{2 / 5} F(q)=\frac{f\left(-q^{1 / 5}\right)}{f\left(-q^{5}\right)} .
$$

Received by the editors February 21, 1997.

1991 Mathematics Subject Classification. Primary 33D10; Secondary 11A55.

Key words and phrases. Rogers-Ramanujan continued fraction, Euler's pentagonal number theorem, Jacobi triple product identity, quintuple product identity.

(C) 1998 American Mathematical Society 
Theorem 1.2. For $|q|<1$,

$$
\frac{1}{F^{5}(q)}-11 q-q^{2} F^{5}(q)=\frac{f^{6}(-q)}{f^{6}\left(-q^{5}\right)}
$$

They were found by Watson [10] in Ramanujan's notebooks. He conjectured that Ramanujan expanded $f\left(-q^{1 / 5}\right)$ and then arranged the terms in the expansion in groups. Watson used the Jacobi triple product identity (2.1), Euler's identity (2.2), Jacobi's identity and the quintuple product identity $[1$, pp. 39, 82$]$ to prove them. Another nice proof was found by Berndt [1, p. 84]. Recently, Berndt, Huang, Sohn and Son [5] found proofs of certain refinements of these identities, which lead to different proofs of (1.1) and (1.2). Berndt, Chan and Zhang [3], [4] employed these identities in their evaluation of $F(q)$ for several values of $q$, including all the values that Ramanujan claimed in his lost notebook.

On page 207 in his lost notebook [8], Ramanujan listed three identities

$$
\begin{gathered}
P-Q=1+\frac{f\left(-q^{1 / 5},-\lambda q^{2 / 5}\right)}{q^{1 / 5} f\left(-\lambda^{10} q^{5},-\lambda^{15} q^{10}\right)}, \\
P Q=1-\frac{f\left(-\lambda,-\lambda^{4} q^{3}\right) f\left(-\lambda^{2} q,-\lambda^{3} q^{2}\right)}{f^{2}\left(-\lambda^{10} q^{5},-\lambda^{15} q^{10}\right)},
\end{gathered}
$$

and

$$
P^{5}-Q^{5}=1+5 P Q+5 P^{2} Q^{2}+\frac{f\left(-q,-\lambda^{5} q^{2}\right) f^{5}\left(-\lambda^{2} q,-\lambda^{3} q^{2}\right)}{q f^{6}\left(-\lambda^{10} q^{5},-\lambda^{15} q^{10}\right)},
$$

without specifying the functions $P$ and $Q$. In this article, the functions $P$ and $Q$ are determined and the identities which are remarkable generalizations of (1.1) and (1.2) are proved. In addition, a useful formula called the Ramanujan product is presented in two distinct forms.

Unexpectedly, it turns out that these claims are evidence that Watson's conjecture is amazingly accurate. Ramanujan indeed had known the simplest way of deriving the identities.

\section{Preliminary Results}

As customary, set

$$
(a ; q)_{\infty}:=\prod_{i=0}^{\infty}\left(1-a q^{i}\right), \quad|q|<1 .
$$

We shall frequently use the following Jacobi triple product identity [1, p. 35].

Theorem 2.1. For $|a b|<1$, we have

$$
f(a, b)=(-a ; a b)_{\infty}(-b ; a b)_{\infty}(a b ; a b)_{\infty} .
$$

By applying (2.1) and simplification, we find that

$$
f(-q)=(q ; q)_{\infty}
$$

which is Euler's pentagonal number theorem.

The following identities are written in Ramanujan's notebooks [1, pp. 48, 34]. 
Theorem 2.2. Let $U_{n}=a^{n(n+1) / 2} b^{n(n-1) / 2}$ and $V_{n}=a^{n(n-1) / 2} b^{n(n+1) / 2}$ for each integer $n$. Then

$$
f(a, b)=\sum_{r=0}^{n-1} U_{r} f\left(\frac{U_{n+r}}{U_{r}}, \frac{V_{n-r}}{U_{r}}\right) .
$$

Theorem 2.3. For $|a b|<1$,

$$
f(a, b)=a^{n(n+1) / 2} b^{n(n-1) / 2} f\left(a(a b)^{n}, b(a b)^{-n}\right) .
$$

On page 207 in his lost notebook, Ramanujan recorded the quintuple product identity in the following form [1, p. 82].

Theorem 2.4. For $\left|\lambda x^{3}\right|<1$,

$$
f\left(-\lambda^{2} x^{3},-\lambda x^{6}\right)+x f\left(-\lambda,-\lambda^{2} x^{9}\right)=\frac{f\left(-x^{2},-\lambda x\right) f\left(-\lambda x^{3}\right)}{f\left(-x,-\lambda x^{2}\right)} .
$$

The Rogers-Ramanujan continued fraction can be expressed in terms of the quotient of theta functions.

Theorem 2.5. For $|q|<1$,

$$
F(q)=\frac{f\left(-q,-q^{4}\right)}{f\left(-q^{2},-q^{3}\right)}, \quad|q|<1 .
$$

The proof follows from the famous Rogers-Ramanujan identities [1, p. 79] which were established for the first time by Rogers in 1894 [9].

\section{The Ramanujan product}

Special cases of Theorem 3.1 can be found in Berndt's books [1, pp. 264, 307, $346,348]$, [2, pp. 142, 145, 188, 192]. We shall use instances of the following general product formula in the proof of (1.5).

Theorem 3.1. For $|a b|<1$, arbitrary integers $j, k$ with $(j, k) \not \equiv(0,0)(\bmod p)$, and a prime $p>2$, let $\zeta:=\exp (2 \pi i / p)$, and $s$ be a solution of

$$
(j+k) x+j \equiv 0 \quad(\bmod p) \quad \text { and } \quad 0 \leq x<p .
$$

Then

$$
\begin{aligned}
& \prod_{n=1}^{p} f\left(\zeta^{j n} a, \zeta^{k n} b\right) \\
& =\left\{\begin{array}{lrr}
\frac{f^{p}\left(a^{s+1} b^{s}, a^{p-s-1} b^{p-s}\right) f\left(a^{p}, b^{p}\right)}{f\left(a^{p(s+1)} b^{p s}, a^{p(p-s-1)} b^{p(p-s)}\right)}, & \text { if } j+k \neq 0 & (\bmod p), \\
f^{p}(-a b) \frac{f\left(a^{p}, b^{p}\right)}{f\left(-a^{p} b^{p}\right)}, & \text { if } j+k \equiv 0 & (\bmod p) .
\end{array}\right.
\end{aligned}
$$

Proof. Let

$$
C:=\prod_{n=1}^{p} f\left(-\zeta^{j n} a,-\zeta^{k n} b\right)
$$

By the Jacobi triple product identity (2.1),

$$
C=\prod_{n=1}^{p}\left(\zeta^{j n} a ; \zeta^{(j+k) n} a b\right)_{\infty}\left(\zeta^{k n} b ; \zeta^{(j+k) n} a b\right)_{\infty}\left(\zeta^{(j+k) n} a b ; \zeta^{(j+k) n} a b\right)_{\infty}
$$


Set

$$
\begin{aligned}
C_{1} & :=\prod_{\ell=1}^{p}\left(\zeta^{j \ell} a ; \zeta^{(j+k) \ell} a b\right)_{\infty}, \\
C_{2} & :=\prod_{\ell=1}^{p}\left(\zeta^{k \ell} b ; \zeta^{(j+k) \ell} a b\right)_{\infty},
\end{aligned}
$$

and

$$
C_{3}:=\prod_{\ell=1}^{p}\left(\zeta^{(j+k) \ell} a b ; \zeta^{(j+k) \ell} a b\right)_{\infty} .
$$

If $j+k \not \equiv 0(\bmod p)$, then

$$
\begin{aligned}
C_{1} & \prod_{\substack{n=0 \\
n \equiv s(\bmod p)}}^{\infty}\left(1-a(a b)^{n}\right)^{p} \prod_{\substack{n=0 \\
n \neq s(\bmod p)}}^{\infty}\left(1-a^{p}(a b)^{p n}\right) \\
= & \prod_{n=0}^{\infty}\left(1-a(a b)^{p n+s}\right)^{p} \prod_{n=0}^{\infty}\left(1-a^{p}(a b)^{p n}\right) / \prod_{\substack{n=0 \\
n \equiv s(\bmod p)}}^{\infty}\left(1-a^{p}(a b)^{p n}\right) \\
= & \left(a^{s+1} b^{s} ; a^{p} b^{p}\right)_{\infty}^{p} \frac{\left(a^{p} ; a^{p} b^{p}\right)_{\infty}}{\left(a^{p(s+1)} b^{p s} ; a^{p^{2}} b^{p^{2}}\right)_{\infty}} .
\end{aligned}
$$

Similarly, as $p-s-1$ is a solution of $(j+k) x+k \equiv 0(\bmod p)$,

$$
C_{2}=\left(a^{p-s-1} b^{p-s} ; a^{p} b^{p}\right)_{\infty}^{p} \frac{\left(b^{p} ; a^{p} b^{p}\right)_{\infty}}{\left(a^{p(p-s-1)} b^{p(p-s)} ; a^{p^{2}} b^{p^{2}}\right)_{\infty}}
$$

and

$$
C_{3}=\left(a^{p} b^{p} ; a^{p} b^{p}\right)_{\infty}^{p} \frac{\left(a^{p} b^{p} ; a^{p} b^{p}\right)_{\infty}}{\left(a^{p^{2}} b^{p^{2}} ; a^{p^{2}} b^{p^{2}}\right)_{\infty}} .
$$

Hence, by the Jacobi triple product identity,

$$
\begin{aligned}
C= & C_{1} C_{2} C_{3} \\
= & \left\{\left(a^{s+1} b^{s} ; a^{p} b^{p}\right)_{\infty}\left(a^{p-s-1} b^{p-s} ; a^{p} b^{p}\right)_{\infty}\left(a^{p} b^{p} ; a^{p} b^{p}\right)_{\infty}\right\}^{p} \\
& \times \frac{\left(a^{p} ; a^{p} b^{p}\right)_{\infty}\left(b^{p} ; a^{p} b^{p}\right)_{\infty}\left(a^{p} b^{p} ; a^{p} b^{p}\right)_{\infty}}{\left(a^{p(s+1)} b^{p s} ; a^{p^{2}} b^{p^{2}}\right)_{\infty}\left(a^{p(p-s-1)} b^{p(p-s)} ; a^{p^{2}} b^{p^{2}}\right)_{\infty}\left(a^{p^{2}} b^{p^{2}} ; a^{p^{2}} b^{p^{2}}\right)_{\infty}} \\
= & f^{p}\left(-a^{s+1} b^{s},-a^{p-s-1} b^{p-s}\right) \frac{f\left(-a^{p},-b^{p}\right)}{f\left(-a^{p(s+1)} b^{p s},-a^{p(p-s-1)} b^{p(p-s)}\right)} .
\end{aligned}
$$

If $j+k \equiv 0(\bmod p)$,

$$
C_{1}=\prod_{n=0}^{\infty}\left(1-a^{p}(a b)^{p n}\right)=\left(a^{p} ; a^{p} b^{p}\right)_{\infty} .
$$

Similarly,

$$
C_{2}=\left(b^{p} ; a^{p} b^{p}\right)_{\infty}
$$


and by $(2.2)$,

$$
C_{3}=(a b ; a b)_{\infty}^{p}=f^{p}(-a b) .
$$

Hence, by the Jacobi triple product identity, we deduce that

$$
C=C_{1} C_{2} C_{3}=f^{p}(-a b)\left(a^{p} ; a^{p} b^{p}\right)_{\infty}\left(b^{p} ; a^{p} b^{p}\right)_{\infty}=f^{p}(-a b) \frac{f\left(-a^{p},-b^{p}\right)}{f\left(-a^{p} b^{p}\right)},
$$

and so the proof is complete after replacing $(-a,-b)$ by $(a, b)$.

As Ramanujan's general theta function $f(a, b)$ has the same generality as the classical theta functions $\theta_{i}(z, q)$, for $i=1,2,3,4$, and they are convertible to each other directly, the Ramanujan product formula (3.1) can be expressed in terms of the classical theta functions. The theta function defined by

$$
\vartheta(z, q):=\theta_{3}(z, q)=\sum_{n=-\infty}^{\infty} q^{n^{2}} e^{2 i n z}, \quad|q|<1,
$$

shall be used in the following theorem.

Theorem 3.2. For $0<|q|<1$ and $z \in \mathbb{C}$, let $j, k, p$ and $s$ be defined as in Theorem 3.1. Then

$$
\begin{aligned}
& \prod_{n=1}^{p} \vartheta\left(z+\frac{\pi n(j-k)}{2 p}, e^{\pi i(j+k) n / p} q\right) \\
& = \begin{cases}\frac{\vartheta^{p}\left(z+\frac{i}{2}(p-2 s-1) \log q, q^{p}\right) \vartheta\left(p z, q^{p}\right)}{\vartheta\left(p z+\frac{i}{2} p(p-2 s-1) \log q, q^{p^{2}}\right)}, & \text { if } \quad j+k \neq 0 \quad \quad(\bmod p), \\
\vartheta^{p}\left(\frac{i}{2} \log q, q^{3}\right) \vartheta\left(p z, q^{p}\right) / \vartheta\left(\frac{i}{2} p \log q, q^{3 p}\right), & \text { if } \quad j+k \equiv 0 \quad(\bmod p) .\end{cases}
\end{aligned}
$$

Proof. Let $a:=q e^{2 i z}$ and $b:=q e^{-2 i z}$. If $A=Q e^{2 i Z}$ and $B=Q e^{-2 i Z}$, equivalently, if $Z=\frac{i}{4} \log (B / A)$ and $Q=\sqrt{A B}$, then by definitions,

$$
f(A, B)=\vartheta(Z, Q),
$$

which are well-defined if $0<|Q|=|\sqrt{A B}|<1$. Let $\zeta:=\exp (2 \pi i / p)$. If $A=-\zeta^{j n} a$ and $B=-\zeta^{k n} b$, then by the above relations,

$$
Z=z+\frac{\pi n(j-k)}{2 p} \text { and } Q=e^{\pi i(j+k) n / p} q .
$$

Thus,

$$
f\left(-\zeta^{j n} a,-\zeta^{k n} b\right)=\vartheta\left(z+\frac{\pi n(j-k)}{2 p}, e^{\pi i(j+k) n / p} q\right)
$$

Similarly,

$$
\begin{aligned}
& f^{p}\left(-a^{s+1} b^{s},-a^{p-s-1} b^{p-s}\right)=\vartheta^{p}\left(z+\frac{i}{2}(p-2 s-1) \log q, q^{p}\right) \\
& f\left(-a^{p},-b^{p}\right)=\vartheta\left(p z, q^{p}\right) \\
& f\left(-a^{p(s+1)} b^{p s},-a^{p(p-s-1)} b^{p(p-s)}\right)=\vartheta\left(p z+\frac{i}{2} p(p-2 s-1) \log q, q^{p^{2}}\right) \\
& f^{p}(-a b)=\vartheta^{p}\left(\frac{i}{2} \log q, q^{3}\right)
\end{aligned}
$$


and

$$
f\left(-a^{p} b^{p}\right)=\vartheta\left(\frac{i}{2} p \log q, q^{3 p}\right) .
$$

Using each of these equalities in (3.1), we achieve the desired result.

\section{Main identities}

Theorem 4.1. If

$$
P=\frac{f\left(-\lambda^{10} q^{7},-\lambda^{15} q^{8}\right)+\lambda q f\left(-\lambda^{5} q^{2},-\lambda^{20} q^{13}\right)}{q^{1 / 5} f\left(-\lambda^{10} q^{5},-\lambda^{15} q^{10}\right)}
$$

and

$$
Q=\frac{\lambda f\left(-\lambda^{5} q^{4},-\lambda^{20} q^{11}\right)-\lambda^{3} q f\left(-q,-\lambda^{25} q^{14}\right)}{q^{-1 / 5} f\left(-\lambda^{10} q^{5},-\lambda^{15} q^{10}\right)}
$$

then (1.3), (1.4) and (1.5) hold.

Proofs. In (2.3), let $a=-q^{1 / 5}, b=-\lambda q^{2 / 5}$, and $n=5$, and then employ (2.4) so as to obtain (1.3).

By (4.1) and (4.2), the identity (1.4) is equivalent to the identity,

$$
\begin{aligned}
S:= & f\left(-\lambda,-\lambda^{4} q^{3}\right) f\left(-\lambda^{2} q,-\lambda^{3} q^{2}\right) \\
= & f\left(-\lambda^{10} q^{5},-\lambda^{15} q^{10}\right) f\left(-\lambda^{10} q^{5},-\lambda^{15} q^{10}\right) \\
& -\lambda f\left(-\lambda^{5} q^{4},-\lambda^{20} q^{11}\right) f\left(-\lambda^{10} q^{7},-\lambda^{15} q^{8}\right) \\
& -\lambda^{2} q f\left(-\lambda^{5} q^{4},-\lambda^{20} q^{11}\right) f\left(-\lambda^{5} q^{2},-\lambda^{20} q^{13}\right) \\
& +\lambda^{3} q f\left(-q,-\lambda^{25} q^{14}\right) f\left(-\lambda^{10} q^{7},-\lambda^{15} q^{8}\right) \\
& +\lambda^{4} q^{2} f\left(-q,-\lambda^{25} q^{14}\right) f\left(-\lambda^{5} q^{2},-\lambda^{20} q^{13}\right) .
\end{aligned}
$$

Let

$$
S=\sum_{u=-\infty}^{\infty} \sum_{v=-\infty}^{\infty} h(u, v)
$$

where

$$
h(u, v):=(-1)^{u+v} \lambda^{\frac{1}{2}\left(5 u^{2}+5 v^{2}-u-3 v\right)} q^{\frac{1}{2}\left(3 u^{2}+3 v^{2}-u-3 v\right)} .
$$

Since

$$
5 u=2(2 u+v)+(u-2 v) \equiv 0 \quad(\bmod 5),
$$

$u-2 v \equiv-2 k(\bmod 5)$ if $2 u+v \equiv k(\bmod 5)$. Write

$$
S=S_{0}+S_{1}+S_{2}+S_{3}+S_{4},
$$

where $S_{k}$ denotes the sum for $2 u+v \equiv k(\bmod 5), 0 \leq k \leq 4$. If $2 u+v \equiv 0$, then $u-2 v \equiv 0$. Let $2 u+v=5 m$ and $u-2 v=-5 n$. Then $u=2 m-n, v=m+2 n$, and

$$
h(u, v)=h(2 m-n, m+2 n)=(-1)^{(3 m+n)} \lambda^{\frac{5}{2}\left(5 m^{2}+5 n^{2}-m-n\right)} q^{\frac{5}{2}\left(3 m^{2}+3 n^{2}-m-n\right)} .
$$


Therefore,

$$
\begin{aligned}
S_{0}= & \sum_{\substack{u, v \\
2 u+v \equiv 0}} h(u, v) \\
= & \sum_{m=-\infty}^{\infty} \sum_{n=-\infty}^{\infty} h(2 m-n, m+2 n) \\
= & \sum_{m=-\infty}^{\infty} \sum_{n=-\infty}^{\infty}(-1)^{(3 m+n)} \lambda^{\frac{5}{2}\left(5 m^{2}+5 n^{2}-m-n\right)} q^{\frac{5}{2}\left(3 m^{2}+3 n^{2}-m-n\right)} \\
= & \sum_{m=-\infty}^{\infty}(-1)^{m}\left(\lambda^{25} q^{15}\right)^{m^{2} / 2}\left(\lambda^{-5} q^{-5}\right)^{m / 2} \\
& \times \sum_{n=-\infty}^{\infty}(-1)^{n}\left(\lambda^{25} q^{15}\right)^{n^{2} / 2}\left(\lambda^{-5} q^{-5}\right)^{n / 2} \\
= & f\left(-\lambda^{10} q^{5},-\lambda^{15} q^{10}\right) f\left(-\lambda^{10} q^{5},-\lambda^{15} q^{10}\right) .
\end{aligned}
$$

Similarly,

$$
\begin{aligned}
& S_{1}=-\lambda f\left(-\lambda^{5} q^{4},-\lambda^{20} q^{11}\right) f\left(-\lambda^{10} q^{7},-\lambda^{15} q^{8}\right), \\
& S_{2}=-\lambda^{2} q f\left(-\lambda^{5} q^{4},-\lambda^{20} q^{11}\right) f\left(-\lambda^{5} q^{2},-\lambda^{20} q^{13}\right), \\
& S_{3}=\lambda^{3} q f\left(-q,-\lambda^{25} q^{14}\right) f\left(-\lambda^{10} q^{7},-\lambda^{15} q^{8}\right),
\end{aligned}
$$

and

$$
S_{4}=\lambda^{4} q^{2} f\left(-q,-\lambda^{25} q^{14}\right) f\left(-\lambda^{5} q^{2},-\lambda^{20} q^{13}\right) .
$$

Substituting (4.5)-(4.9) in (4.4) and then using (4.3), we complete the proof of (1.4).

Let $\zeta=\exp (2 \pi i / 5)$. In (1.3), replace $q^{1 / 5}$ by $\zeta^{n} q^{1 / 5}$ for $n=1,2,3,4,5$, and then multiply the five identities. Then we find that

$$
\prod_{n=1}^{5}\left(\frac{P}{\zeta^{n}}-\zeta^{n} Q-1\right)=\frac{1}{q f^{5}\left(-\lambda^{10} q^{5},-\lambda^{15} q^{10}\right)} \prod_{n=1}^{5} f\left(-\zeta^{n} q^{1 / 5},-\zeta^{2 n} \lambda q^{2 / 5}\right) .
$$

Simplifying the left side of (4.10) yields

$$
P^{5}-Q^{5}-1-5 P Q-5 P^{2} Q^{2} .
$$

In Theorem 3.1, let $j=1, k=2, s=3, a=-q^{1 / 5}, b=-\lambda q^{2 / 5}$ and $p=5$. Then

$$
\prod_{n=1}^{5} f\left(-\zeta^{n} q^{1 / 5},-\zeta^{2 n} \lambda q^{2 / 5}\right)=\frac{f\left(-q,-\lambda^{5} q^{2}\right) f^{5}\left(-\lambda^{2} q,-\lambda^{3} q^{2}\right)}{f\left(-\lambda^{10} q^{5},-\lambda^{15} q^{10}\right)}
$$

Using (4.11) in (4.10), we finish the proof of (1.5).

Now we shall show that (1.1) and (1.2) are special cases of (1.3) and (1.5).

Proofs of (1.1) and (1.2). Let $\lambda=1$ in (1.3) and (1.5). Then by applying the quintuple identity $(2.5)$ with $(x, \lambda)=\left(q, q^{2}\right)$ and $\left(q^{2}, q^{-1}\right)$, respectively, we see that, by (2.4) and (2.6),

$$
P=\frac{f\left(-q^{7},-q^{8}\right)+q f\left(-q^{2},-q^{13}\right)}{q^{1 / 5} f\left(-q^{5}\right)}=\frac{f\left(-q^{2},-q^{3}\right)}{q^{1 / 5} f\left(-q,-q^{4}\right)}=\frac{1}{q^{1 / 5} F(q)},
$$


and

$$
Q=\frac{f\left(-q^{4},-q^{11}\right)-q f\left(-q,-q^{14}\right)}{q^{-1 / 5} f\left(-q^{5}\right)}=\frac{q^{1 / 5} f\left(-q,-q^{4}\right)}{f\left(-q^{2},-q^{3}\right)}=q^{1 / 5} F(q) .
$$

Since $P Q=1,(1.3)$ and (1.5) become (1.1) and (1.2).

\section{ACKNOWLEDGMENT}

The author would like to express deep appreciation to Professor Bruce C. Berndt for his warm encouragement and invaluable suggestions.

\section{REFERENCES}

1. B. C. Berndt, Ramanujan's Notebooks, Part III, Springer-Verlag, New York, 1991. MR 95j:01069

2. B. C. Berndt, Ramanujan's Notebooks, Part IV, Springer-Verlag, New York, 1994. MR 95e: 11028

3. B. C. Berndt and H. H. Chan, Some values for the Rogers-Ramanujan continued fraction, Canadian J. Math. 47 (1995), 897-914. MR 97a:33043

4. B. C. Berndt, H. H. Chan and L.-C. Zhang, Explicit evaluations of the Rogers-Ramanujan continued fraction, J. Reine Angew. Math. 480 (1996), 141-159. CMP 97:04

5. B. C. Berndt, S.-S. Huang, J. Sohn and S. Son, Some theorems on the Rogers-Ramanujan continued fraction in Ramanujan's lost notebook (preprint).

6. B. C. Berndt and R. A. Rankin, Ramanujan: Letters and Commentary, Amer. Math. Soc., Providence, 1995. MR 97c:01034

7. S. Ramanujan, Notebooks (2 volumes), Tata Institute of Fundamental Research, Bombay, 1957. MR 20:6340

8. S. Ramanujan, The Lost Notebook and Other Unpublished Papers, Narosa, New Delhi, 1988. MR 89j:01078

9. L. J. Rogers, Second memoir on the expansion of certain infinite products, Proc. London Math. Soc. 25 (1894), 318-343.

10. G. N. Watson, Theorems stated by Ramanujan (VII): Theorems on continued fractions, J. London Math. Soc. 4 (1929), 39-48.

11. E. T. Whittaker and G. N. Watson, A Course of Modern Analysis, 4 ed., University Press, Cambridge, 1996. MR 97k:01072

Department of Mathematics, University of Illinois at Urbana-Champaign, 1409 W. Green St., Urbana, Illinois 61801

E-mail address: son@math.uiuc.edu 